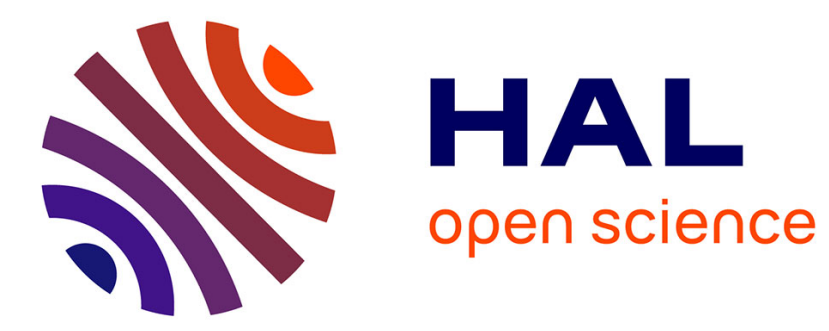

\title{
Positional Scoring Rules with Uncertain Weights
}

Paolo Viappiani

\section{To cite this version:}

Paolo Viappiani. Positional Scoring Rules with Uncertain Weights. SUM 2018: Scalable Uncertainty Management, Oct 2018, Milan, Italy. pp.306-320, 10.1007/978-3-030-00461-3_21 . hal-01983344

\section{HAL Id: hal-01983344 https://hal.sorbonne-universite.fr/hal-01983344}

Submitted on 16 Jan 2019

HAL is a multi-disciplinary open access archive for the deposit and dissemination of scientific research documents, whether they are published or not. The documents may come from teaching and research institutions in France or abroad, or from public or private research centers.
L'archive ouverte pluridisciplinaire $\mathbf{H A L}$, est destinée au dépôt et à la diffusion de documents scientifiques de niveau recherche, publiés ou non, émanant des établissements d'enseignement et de recherche français ou étrangers, des laboratoires publics ou privés. 


\title{
Positional Scoring Rules with Uncertain Weights ${ }^{\star}$
}

\author{
Paolo Viappiani ${ }^{1}$ \\ Sorbonne Université, UMR7606 CNRS, LIP6, 4 pl. Jussieu, 75005 Paris, France \\ paolo.viappiani@lip6.fr
}

\begin{abstract}
Positional scoring rules are frequently used for rank aggregation (for example in social choice and in sports). These rules are highly sensitive to the weights associated to positions: depending on the weights, a different winner may be selected. In this paper we explicitly consider the role of weight uncertainty in both the case of monotone decreasing weights and of convex decreasing weights. First we discuss the problem of finding possible winners (candidates that may win for a feasible instantiation of the weights) based on previous works that established a connection with the notion of stochastic dominance. Second, we adopt decision-theoretic methods (minimax regret, maximum advantage, expected value) to pick a winner based on the weight uncertainty and we provide a characterization of these methods. Finally, we show some applications of our methodology in real datasets.
\end{abstract}

Keywords: Scoring rules $\cdot$ rank aggregation $\cdot$ possible winners $\cdot$ minimax regret $\cdot$ stochastic dominance $\cdot$ convex sequences

\section{Introduction}

In many contexts it is necessary to aggregate several rankings and either pick a winner or determine an output ranking. For example, rank aggregation emerges in recommender systems and social choice (preference aggregation), in information retrieval (aggregation of the output of several search engines), in sports (aggregation of the performance in several races into a single score). Positional scoring rules are frequently used due to their simplicity; in addition they satisfy a number of interesting properties (one of the most prominent results is that of Young [14]: a symmetric social choice function is continuous and consistent if and only if it is a scoring rule). Among positional scoring rules, Borda is a well-known method whose properties have been studied in depth $[13,4]$.

A scoring rule assigns a scores to each candidate based on the rank obtained in each ranking. The output of a scoring rule crucially depends on the weights (attached to ranks); in general several different winners are possible with different weights. In this article we consider methods to generate a winner using positional scoring rules under weight uncertainty. We assume non increasing weights; we also discuss the case of scoring rules where weights constitute a convex sequence.

* Work supported by the ANR project Cocorico-CoDec (ANR-14-CE24-0007-01). 
We assume $n$ agents express preferences in the form of rankings involving a set of $m$ candidates $A=\{a, b, c, \ldots\}$; rankings are assumed to be linear orders (complete, transitive, asymmetric and irreflexive binary relation). Positional scoring rules discriminate between candidates by assigning a fixed score to each rank. Scoring rules assign a score to each alternative based on its rank distribution; let $v_{j}^{x}$ be the number of times alternative $x$ was ranked in the $j$-th position. Note that $\sum_{j=1}^{m} v_{j}^{x}=n$ for each $x \in A$ and $\sum_{x \in A} v_{j}^{x}=n$ for each $j=1, \ldots, m$.

A scoring rule specifies the vector of weights $w_{1}, \ldots, w_{m}$ (also called scoring vector) to be assigned to each position. The score obtained by a candidate according to weight vector $w=\left(w_{1}, \ldots, w_{m}\right)$ is $s_{w}(x)=\sum_{j=1}^{m} w_{j} v_{j}^{x}$. These total scores can be used to pick a winner or to rank the alternatives. For example plurality is obtained by setting $w_{1}=1$ and $w_{j}=0$ for all $j \in\{2, \ldots, m\}$.

By choosing a particular $w$, it is possible to specify some preferences on which kind of aggregation is desired, by giving more or less weight to the first positions compared to the positions that came afterwards in the ranking. First of all, we assume that not all weights are null, otherwise the alternatives are not discriminated (degenerated scoring rule). A natural hypothesis ${ }^{1}$, that we adopt here, is to require that the sequence of weights is non-increasing: $w_{i} \geq w_{i+1}$ for all $i \in\{1, \ldots, m-1\}$; intuitively, in a ranking an alternative is (weakly) preferred to the alternatives that comes afterwards in the ranking.

A scoring rule is invariant to affine positive transformation of the scoring vector, that means that ranking the $w^{\prime}=\alpha w+\beta$ with $\alpha>0$ and arbitrary $\beta$ gives the same output. Therefore, with no loss of generality, we let $w_{1}=1$ and $w_{m}=0$ (therefore we have $m-2$ degrees of freedom). Given this assumption the Borda rule is given by setting $w_{j}=\frac{m-j}{m-1}$.

Moreover, it is often (but not always) assumed in practice that the positional weights constitute a convex sequence, meaning that the difference between the first and the second weight is not less than the difference between the second and of the third, and so on. In such a case the weights need to satisfy the following constraint, for each $i$ between 1 and $m-2$ :

$$
w_{i}-w_{i+1} \geq w_{i+1}-w_{i+2} \Longleftrightarrow w_{i}-2 w_{i+1}+w_{i+2} \geq 0
$$

Note that Borda and plurality are convex; furthermore convexity is often satisfied by the weights used when combining ranks in sports, races and other situations (e.g. formula one world championship, alpine skiing world cup).

We argue that setting a precise vector of weights for a scoring rule can be seen arbitrary; indeed the decision of which weights to use is critical since different weights often lead to different winners. Therefore there is interest to reason not just with a fixed vector $w$ but about possible sets of parameters.

Notation We introduce some of the notation that we will use in the paper. We use $\llbracket m \rrbracket$ to denote the set $\{1, \ldots, m\}$. Given two vectors $v^{1}$ and $v^{2}$ we write

\footnotetext{
${ }^{1}$ This hypothesis is removed in Goldsmith et al. [5] where the authors allow preferences for intermediate positions.
} 
$v^{1} \succeq v^{2}$ iff $v_{j}^{1} \geq v_{j}^{2}$ for all components $j$. If the inequalities are strict, $v_{j}^{1}>v_{j}^{2}$ for all components $j$, then we write $v^{1} \succ v^{2}$.

We will use $W^{D}$ to denote the set of scoring vectors with non-increasing weights and $W^{C}$ to denote the set of non-increasing (i.e. weakly decreasing) scoring vectors whose weights constitute a convex sequence ${ }^{2}$ (with our boundary assumptions, $w_{1}=1$ and $\left.w_{m}=0\right)$ :

$$
\begin{aligned}
& W^{D}=\left\{\left(w_{1}, \ldots, w_{m}\right) \mid 1=w_{1} \geq w_{2} \geq \ldots \geq w_{m-1} \geq w_{m}=0\right\} \\
& W^{C}=\left\{\left(w_{1}, \ldots, w_{m}\right) \mid w \in W^{D} \wedge w_{i}-2 w_{i+1}+w_{i+2} \geq 0 \quad \forall i \in \llbracket m-2 \rrbracket\right\} .
\end{aligned}
$$

\section{Dominance and Possible Winners}

In this section we discuss dominance relations and possible winners. The first step is to reformulate scoring rules in terms of cumulative ranks. This is useful in order to establish dominance relations between alternatives in the context of scoring rules. Dominance [11] holds between alternative $x$ and alternative $y$ iff the former has higher score than the latter alternative for any possible scoring rule; $y$ is then said to be dominated. A rational decision maker will then never choose a dominated alternative; since, no matter how the weights are defined, there is another alternative that is at least as good (in case of weak dominance) or strictly better (strict dominance).

We will then refine undominated alternatives in order to identify which candidates are possible winners.

\subsection{Reformulation using Cumulative Standings}

We consider cumulative standings that represent the fraction of times that a candidate was ranked over a certain point. Cumulative standings are defined as the cumulative sum of the rank vectors, starting from the first position. The vector $V^{x}=\left(V_{1}^{x}, \ldots, V_{m-1}^{x}\right)$ is such that $V_{j}^{x}=\sum_{l=1}^{j} v_{l}^{x}$ is the number of times that alternative $x$ has been ranked in position $j$ or better. Note that we consider in $V^{x}$ only $m-1$ components: indeed $V_{m}^{x}$, the number of cumulative standings in the last place, would always equal to the number of voters $n$.

We now define the vector $\delta=\left(\delta_{1}, \ldots, \delta_{m-1}\right)$, dubbed differential weights, as the vector of the differences between two successive positional weights of the original scoring vector:

$$
\delta_{j}=w_{j}-w_{j+1} \quad \forall j \in \llbracket m-1 \rrbracket .
$$

Remember that we assumed that $w_{1}=1$ and $w_{m}=0$; this assumption means that we have $\delta_{1}=1-w_{2}$ and $\delta_{m-1}=w_{m-1}$. The score obtained by an alternative

\footnotetext{
2 There is some redundancy in the constraints: it is enough to assume convexity and $w_{m-1} \geq 0$ to ensure that the sequence is not increasing.
} 
$x$ can now be expressed in function of $\delta$ and the cumulative standings $V$ :

$$
s_{\delta}(x)=\sum_{j=1}^{m-1} \delta_{j} V_{j}^{x}
$$

where we use the subscript $\delta$ to underline the dependency on the differential weight. Decreasing weights in the original formulation correspond to positive differential weights. Note that the original rank vectors can be expressed in terms of cumulative standings: $v_{j}=V_{j}-V_{j-1}$; similarly the original weights can be recovered from the differential weights: $w_{j}=\sum_{l=j}^{m-1} \delta_{l}$. The requirement that the first weight should be equal to one can therefore be written as: $\sum_{j=1}^{m} \delta_{j}=1$ (and this indirectly bounds all other weights to be lower than 1 due to monotonicity). The score of an alternative can therefore be seen as a convex combination of the cumulative ranks.

We now derive a reformulation that is useful for scoring vectors that constitute convex sequences. To do so we need to introduce the vector of cumulative of the cumulative standings:

$$
\mathcal{V}_{j}^{x}=\sum_{l=1}^{j} V_{l}^{x}=\sum_{l=1}^{j} \sum_{o=1}^{l} v_{o}^{x}=\sum_{l=1}^{j}(j-l+1) v_{l}^{x} \quad j \in \llbracket m \rrbracket
$$

We now define a new vector of parameters $\phi$ that represents the convexity of the weights $w$ :

$$
\begin{cases}\phi_{j}=\delta_{j}-\delta_{j+1} & =w_{j}-2 w_{j+1}+w_{j+2} \quad j \in \llbracket m-2 \rrbracket \\ \phi_{m-1}=\delta_{m-1}=w_{m-1} .\end{cases}
$$

Given that we assumed $w_{1}=1$, we have $\phi_{1}=1-2 w_{2}+w_{3}$ and, since $w_{m}=0$, we have $\phi_{m-2}=\delta_{m-2}-\delta_{m-1}=w_{m-2}-2 w_{m-1}$. Stating that $\phi_{j} \geq 0$ for all components $j$ is equivalent to require the weight vector $w$ to be convex.

The score $s_{w}(x)$ of an alternative $x$ under a scoring rule with weight vector $w$ can now be expressed as $\sum_{j=1}^{m-1} \phi_{j} \mathcal{V}_{j}^{x}$ with $\phi$ obtained from $w$ using Equation 4. The constraint that all weights should be bounded and the highest weight, $w_{1}$ has value equal to one, becomes, in terms of convex weights, in assuming that $\sum_{l=1}^{m-1} l \phi_{l}=1$.

\begin{tabular}{|c|c|c|c|}
\hline rule & positional weights & differential weights & convex weights \\
\hline Plurality & $w=(1, \underbrace{0, \ldots, 0})$ & $\delta=(1,0, \ldots, 0)$ & $\phi=(1,0, \ldots, 0)$ \\
\hline$k$-approval & $w=(\underbrace{1, \ldots, 1}, \underbrace{0, \ldots, 0})$ & $\delta=(\underbrace{0, \ldots, 0}, 1, \underbrace{0, \ldots, 0})$ & $\phi=(\underbrace{0, \ldots, 0},-1,1, \underbrace{m-2, \ldots, 0)}$ \\
\hline & $\underbrace{}_{k} \quad \underbrace{}_{m-k}$ & $\overbrace{k-1} \quad \overbrace{m-k-1}$ & $\overbrace{m-k-2}$ \\
\hline Borda & $w=\left(1, \frac{m-2}{m-1}, \frac{m-3}{m-1}, \ldots, 0\right)$ & $\delta=\left(\frac{1}{m-1}, \ldots, \frac{1}{m-1}\right)$ & $\phi=(\underbrace{0, \ldots, 0}_{m-2}, \frac{1}{m-1})$ \\
\hline top-k Bord & $w=(1, \frac{k-2}{k-1}, \ldots, \frac{1}{k-1}, \underbrace{0, \ldots, 0}_{m-k})$ & $\delta=(\underbrace{\frac{1}{k}, \ldots, \frac{1}{k}}_{k}, \underbrace{0, \ldots, 0}_{m-k-1})$ & $\phi=(\underbrace{0, \ldots, 0}_{k-1}, \frac{1}{k}, \underbrace{0, \ldots, 0}_{n-k-1})$ \\
\hline
\end{tabular}

We now show, in the following table, how some common scoring rules are expressed in terms of differential weights and in terms of convex weights. 
We highlight the following observations:

$-k$-approval is not convex when $k \geq 2$. The $k$-approval score of alternative $x$ is exactly $V_{k}^{x}$.

- we call top-K Borda the scoring rule based on Borda restricted to the top $k$ positions. The score of an alternative $x$ with respect to top-k Borda is $\frac{\mathcal{V}_{k}^{x}}{k}$.

\subsection{Dominance relations}

The usefulness of the reformulations presented in Section 2.1 is that they can be used to discriminate candidates according to dominance relations, that allow to identify candidates that are less preferred than another one for any feasible scoring vector. Note that dominance only gives us with a partial order, so it is usually not enough to unambiguously define a winner.

Non-increasing weights When dealing with scoring vectors in $W^{D}$, i.e. with non increasing sequences of weights the set of possible scores obtained by a candidate $x$, with cumulative ranks $V^{x}$, are given by

$$
\left\{\sum_{j=1}^{m-1} \delta_{j} V_{j}^{x} \mid \delta_{1} \geq 0, \ldots, \delta_{m} \geq 0 \wedge \sum_{j=1}^{m} \delta_{j}=1\right\} .
$$

Basically, all convex combinations of the components of $V^{x}$ are possible. Since all elements of $\delta$ are non-negative, we can compare the cumulative ranks of two candidates componentwise to check if a dominance relation exists.

Proposition 1 [11] If $V^{x} \succeq V^{y}$ then the score $x$ is necessarily at least as good than $y$ for any scoring rule with non-increasing weights (and $x$ is necessarily strictly better than $x$ when $\left.V^{x} \succ V^{y}\right)$ :

- $V^{x} \succeq V^{y} \Longrightarrow s_{w}(x) \geq s_{w}(y) \forall w \in W^{D}$,

- $V^{x} \succ V^{y} \Longrightarrow s_{w}(x)>s_{w}(y) \forall w \in W^{D}$.

We say that $x$ weakly dominates $y$ in the first case, and that $x$ strongly dominates $y$ in the second case.

The previous statement can be seen as a form of first-order stochastic dominance. A candidate is said dominated if there exists another candidate that dominates the former.

Convex weights Assuming a non-increasing convex sequence (the scoring vector belongs to $W^{C}$ ), the space of possible scores associated to an alternative is given by

$$
\left\{\sum_{j=1}^{m-1} \phi_{j} \mathcal{V}_{j} \mid \phi_{1} \geq 0, \ldots, \phi_{m} \geq 0 \wedge \sum_{j=1}^{m-1} j \phi_{j}=1\right\} .
$$

All $\phi_{j}$ are non-negative since the sequence is convex. If each element of the vector $\mathcal{V}^{x}$ is at least as big as the corresponding element of $\mathcal{V}^{y}$, than $x$ has at least the same score of $y$ for any scoring rule with convex weight (and the analogous relation holds with strict inequalities). 
Proposition 2 [11] If $\mathcal{V}^{x} \succeq \mathcal{V}^{y}$, then $x$ is at least as good than $y$, for any scoring rule with a convex sequence of decreasing weights (and $x$ is necessarily strictly better than $x$ when $\left.\mathcal{V}^{x} \succ \mathcal{V}^{y}\right)$ :

$-\mathcal{V}^{x} \succeq \mathcal{V}^{y} \Longrightarrow \forall w \in W^{C} \quad s_{w}(x) \geq s_{w}(y)$,

$-\mathcal{V}^{x} \succ \mathcal{V}^{y} \Longrightarrow \forall w \in W^{C} \quad s_{w}(x)>s_{w}(y)$.

Again, the first case is referred as weak dominance and the second as strong dominance.

This is akin to second order stochastic dominance, but considering convex and not concave utility.

Example 1. Consider the following numeric example.The first table reports the distribution of the ranks, the second the cumulative ranks and the third the double cumulative ranks.

\begin{tabular}{|c|c|c|c|c|c|c|c|c|c|c|}
\hline \multicolumn{4}{|c|}{ Candidate $v_{1}^{\circ} v_{2}^{\circ} v_{3}^{\circ} v_{4}^{\prime}$} & \multicolumn{4}{|c|}{ Candidate $V_{1} V_{2} V_{3}$} & \multicolumn{3}{|c|}{ Candidate $\mathcal{V}_{1} \mathcal{V}_{2} \mathcal{V}_{3}$} \\
\hline a & 2 & 2 & 2 & a & 2 & 4 & 6 & $\mathrm{a}$ & 2 & \\
\hline $\mathrm{b}$ & 0 & 6 & 2 & $b$ & 0 & 6 & 8 & $\mathrm{~b}$ & 0 & \\
\hline c & 2 & 0 & 4 & c & 2 & 2 & 6 & c & 2 & 410 \\
\hline d & 4 & 0 & 04 & d & 4 & 4 & 4 & d & 4 & $\begin{array}{ll}8 & 12\end{array}$ \\
\hline
\end{tabular}

- When considering monotone weights, one can establish dominance by pairwise comparisons of rows in the table of cumulative ranks; for instance $a$ weakly-dominates $c$ since $V_{1}^{a}=V_{1}^{c}, V_{2}^{a}>V_{2}^{c}$, and $V_{3}^{a}=V_{3}^{c}$. The set of weakly-undominated candidates is then $\{a, b, d\}$. No strong domination holds.

- When considering convex weights, now $d$ weakly dominates $a$; moreover $d$ strongly dominates $c$ since $V_{j}^{d}>V_{j}^{c}$ for all $j \in \llbracket m-1 \rrbracket$. The set of weakly undominated candidates is $\{b, d\}$, while the set of strictly undominated is $\{a, b, d\}$.

\subsection{Possible Winners}

In the following we present the notions of possible and necessary winners under different assumptions about the scoring vector. The possible winners are all those candidates that may be winners under a realization of the weights; a necessary winner (if it exists) is a winner under any possible weight. Possible winners (and necessary winners) need to be undominated according to the relations described in the previous section. However it is important to note that there might be undominated alternatives that may not be a possible winner.

In what follows, let let $W$ be the set of feasible weights; we focus on $W$ being either $W^{C}$ or $W^{D}$.

Definition 1. A candidate $x$ is a possible co-winner iff there is a weight vector $w$ such that the score of $x$ under $w$ is higher or equal than the score of all other candidates.

$$
\exists w \in W: s_{w}(x) \geq s_{w}(y) \quad \forall y \in A
$$


If the above formula holds with a strict inequality sign, $x$ is a possible winner.

Definition 2. A candidate $x$ is a necessary co-winner iff for all weight vectors $w \in W$ the score of $x$ under $w$ is higher or equal than the score of all other candidates.

$$
\forall w \in W: s_{w}(x) \geq s_{w}(y) \forall y \in A
$$

If the above holds with a strict inequality sign, $x$ is a necessary winner.

Possible winners are a subset of maximal elements of the dominance relations seen in the previous sections; indeed a dominated candidate (in either sense, weak or strong) cannot be a possible winner for any scoring rule. A candidate that is weakly dominated by another candidate but it is not strongly dominated may be a possible co-winner. Moreover, note that if there is only a single candidate that is undominated, then it is a necessary winner.

We show that it may occur that an undominated alternative is not a possible winner with an example.

Example 2. Assume the rank distributions $(n=12, m=3)$ associated to three candidates $a, b, c$ presented in the following table (to be read as follows: $a$ is ranked first 4 time, second 3 times and 5 times last).

\begin{tabular}{llll}
\hline & & & \\
$v_{1}$ & $v_{2}$ & $v_{3}$ \\
\hline a & 4 & 3 & 5 \\
b & 6 & 0 & 6 \\
c & 2 & 9 & 1 \\
\hline
\end{tabular}

As usual we let $w_{1}=1$ and $w_{3}=0$, so the only free parameter is $w_{2}$. The score of $a$ is $s(a)=4+3 w_{2}$; the score of $b$ is $s(b)=6$ and the score of $c$ is $s(c)=2+9 w_{2}$. Can $a$ be a winner for some values of $w_{2}$ ? The answer is no: for $a$ to be better than $b$, we need $w_{2} \geq \frac{2}{3}$ but for $a$ to be better than $c, w_{2}$ should be less than $\frac{1}{3}$.

The cumulative ranks are $V^{a}=(4,7), V^{b}=(6,6), V^{c}=(2,11)$ and no pairwise domination holds between $a, b$, or $c$. Therefore $a$ is neither dominated by $b$ or $c$ but is not a possible winner (and not even a co-winner).

This kind of reasoning has been discussed in [1], dealing with combinatorial problems; we now present similar techniques for computing possible winners.

Computation of Possible Winners We test whether candidate $x$ is a possible winner by finding the maximum difference between its score and that of the best ranked alternative other than $x$. If this is positive, then candidate $x$ is a possible winner, if it is zero is only a possible co-winner; otherwise it is not a possible winner. Formally, define the maximum advantage or margin $\mathrm{MA}(x)$ of $x$ by:

$$
\begin{aligned}
\operatorname{MA}(x) & =\max _{w \in W} \min _{y \neq x} s_{w}(x)-s_{w}(y)=\max _{w \in W} \min _{y \neq x} \sum_{j=1}^{m} w_{j} v_{j}^{x}-\sum_{j=1}^{m} w_{j} v_{j}^{y} \\
& =\max _{w \in W}\left\{\sum_{j=1}^{m} w_{j} v_{j}^{x}-\max _{y \neq x} \sum_{j=1}^{m} w_{j} v_{j}^{y}\right\}
\end{aligned}
$$


where $W \in\left\{W^{D}, W^{C}\right\}$ is the set of possible weight vectors (either the class of non-increasing or the class of convex weights). This can be achieved by the following optimization:

$$
\begin{array}{ll}
\max _{Z, w} & Z \\
\text { s.t. } & Z \leq \sum_{j=1}^{m} w_{j} v_{j}^{x}-\sum_{j=1}^{m} w_{j} v_{j}^{y} \quad \forall y \in A-\{x\} \\
& w_{j} \geq w_{j+1} \\
& w_{j}-2 w_{j+1}+w_{j+2} \geq 0 \quad \forall j \in \llbracket m-2 \rrbracket \\
& w_{1}=1 ; w_{m}=0
\end{array}
$$

Equations 7-11 represent a linear program that can be solved with standard optimization tools such as CPLEX or Gurobi. The alternative $x$ is given in input. There are $m-1$ decision variables, of which $m-2$ represent the scoring vector (we have $m-2$ degrees of freedom, since we assume $w_{1}=1$ and $w_{m}=0$ ), and an additional decision variable $Z$ (representing the margin) whose value is constrained (equation 8 ) to be less than the difference in score between the score of $x$ and any other alternative $y \in A$. The resulting value of $Z$ gives us the best margin with $x$ being the winner when choosing $w$ in $W$. If this value is positive

\begin{tabular}{|c|c|c|c|c|}
\hline \multicolumn{2}{|c|}{ Candidate $v_{1} v_{2} v_{3}^{*} v_{4}$} & \multicolumn{3}{|c|}{ Candidate $\mathrm{MA}_{W^{D}} \mathrm{MA}_{W^{C}}$} \\
\hline $\mathrm{a}$ & $\begin{array}{llll}2 & 2 & 2 & 2\end{array}$ & $\mathrm{a}$ & 0 & -0.29 \\
\hline b & $\begin{array}{llll}0 & 6 & 2 & 0\end{array}$ & b & 2 & 0.66 \\
\hline $\mathrm{c}$ & $\begin{array}{llll}2 & 0 & 4 & 2\end{array}$ & c & 0 & -0.86 \\
\hline d & $\begin{array}{llll}4 & 0 & 0 & 4\end{array}$ & d & 2 & 2 \\
\hline
\end{tabular}
then $x$ is a possible winner. Constraint 10 refers to convex weights (region $W^{C}$ ) and should be removed when dealing with non-increasing weights (region $W^{D}$ ).

Example 3. Consider again the values of Example 1. The maximum advantage is given in the second table, once computed w.r.t. $W^{D}$ and once w.r.t. $W^{C}$.

Note that when considering convex weights, we reduce the feasibility region and therefore the maximum margin will be less. Candidates $b, d$ are possible winners in both cases, since they are associated with a positive maximum advantage. Note that even if $a$ was undominated in $W^{D}$, it is not a possible winner; in $W^{D}$ $a$ and $c$ are possible co-winners for scoring weight $w=(1,0.5,0.5,0)$.

\section{Aggregation of scoring rules with uncertain weights}

In general there may be many possible winners, it is therefore often necessary to have a method to pick a single winner. We now discuss how to adapt classic criteria for decision-making under uncertainty for the case of scoring rules with uncertain weights. 
Maximin and maximax Selecting the alternative according to maximin (criterion that picks the alternative whose worst score is highest) corresponds to setting each weight to zero, except $w_{1}$ that is equal to 1 by assumption; therefore this case corresponds to plurality. Instead maximizing the maximum possible score $^{3}$ (that means using maximax decision rule) corresponds to setting each weight to one, except $w_{m}$ that is set to 0 by assumption; this case corresponds to $(m-1)$ approval for the case of non-increasing scoring rules. For convex rules, maximax is attained by Borda. Of course, these methods are trivial and do not actually support the idea that we should possibly consider a variety of scoring vectors (in $W^{m}$ or in $W^{c}$ depending on the case) for evaluating different candidates.

Minimax regret Minimax regret is a robust decision criterion classically used for optimization under uncertainty $[10,6]$; it has been more recently advocated to be used in decision-making where the uncertainty is over utility values $[2,9]$. In the context of voting, it has been proposed by $\mathrm{Lu}$ and Boutilier [8] as a way to deal with partially elicited rankings (but they assume that the social choice function is known precisely).

We consider now using minimax regret to identify a candidate (to be declared winner) in face of uncertainty over the values of the scoring vector $w_{1}, \ldots, w_{m}$. The idea is to associate each alternative with the maximum loss incurred in terms of score points (with respect to the "true" winner) assuming that an adversary can freely set the scoring vector; the minimax-regret alternative is the one that minimizes such loss. The max regret of alternative $x$ is:

$$
\begin{aligned}
\operatorname{MR}(x) & =\max _{w \in W} \max _{y \in A} s_{w}(y)-s_{w}(x)=\max _{w \in W} s_{w}^{*}-s_{w}(x) \\
& =\max _{w \in W}\left[\max _{y \in A} \sum_{j=1}^{m} w_{j} v_{j}^{y}\right]-\sum_{j=1}^{m} w_{j} v_{j}^{x}
\end{aligned}
$$

where $W$ is either $W^{D}$ (decreasing weights) or $W^{C}$ (convex weights), depending on the context. We then pick the alternative with minimum max regret: $\arg \min _{x \in A} Z^{\mathrm{MR}}(x)$; whatever the weight in $W$, the picked alternatives is at most $\operatorname{MR}(x)$ score points from optimality.

We now show that we can characterize the minimax regret alternative in a way that it is not necessary to solve an optimization problem. As before, we slice out our analysis dealing with 1) non-decreasing weights and 2) convex sequences. Before presenting our results note that the winner according to $k$ approval is then just the alternative with highest value $V_{k}$ (number of times $x$ was ranked in position $k$ or better); let $V_{k}^{*}=\max _{x \in A} V_{k}^{x}$ be such value. Similarly let $\mathcal{V}_{k}^{*}=\max _{x \in A} \mathcal{V}_{k}^{x}$ be the maximum value of the double cumulative ranks for a given position $j$.

\footnotetext{
${ }^{3}$ Several authors, including [3], have proposed to take a similar optimistic approach, although the way the feasible set is defined makes the resulting rules quite different.
} 
Proposition 3 In the case of non-increasing weights:

$$
\operatorname{MR}(x)=\max _{j=1}^{m-1} V_{j}^{*}-V_{j}^{x}
$$

instead, assuming convex weights:

$$
\operatorname{MR}(x)=\max _{j=1}^{\max } \frac{\mathcal{V}_{j}^{*}-\mathcal{V}_{j}^{x}}{j}
$$

Proposition 3 allows $^{4}$ us to compute max regret in an efficient way without solving an optimization problem. While in the original formulation of maximum regret (Equation 13) the maximization is over a continuum of values (all feasible scoring vectors $w$ ), Equations 14 and 15 compute the maximum among a fixed number of alternatives (in the two cases, monotone and convex).

For non-increasing scoring vectors, note that that, since $V_{j}^{*}$ is equivalent to the best score according to $j$-approval, the term $V_{j}^{*}-V_{j}^{x}$ is the loss occurred to $x$ when considering $j$-approval; max regret can be seen as the maximum loss occurred by $x$ with respect to the family of $k$-approval voting rules. The minimax regret optimal alternative is then the candidate that is the least far away from the optimal score attained with any $k$-approval voting rule.

Example 4. We provide an example of max regret computation using the first case of Proposition 3. One needs to consider the table of the cumulative standings; the max-regret of a given candidate is the the maximum shortfall between the values in the candidate's row compared to the best value (in bold below) in

\begin{tabular}{|c|c|c|c|c|c|c|c|c|}
\hline \multicolumn{3}{|c|}{ Candidate $v_{1} v_{2} v_{3} v_{4}$} & \multicolumn{4}{|c|}{ Candidate $V_{1} V_{2}^{\cdot} V_{3}$} & \multicolumn{2}{|c|}{ Candidate $\mathrm{MR}_{W^{D}}$} \\
\hline $\mathrm{a}$ & 22 & 22 & $\mathrm{a}$ & 2 & 4 & 6 & $\mathrm{a}$ & 2 \\
\hline b & $\begin{array}{ll}0 & 6\end{array}$ & 20 & $\mathrm{~b}$ & 0 & 6 & 8 & b & 4 \\
\hline $\mathrm{c}$ & 20 & 42 & $\mathrm{c}$ & 2 & 2 & 6 & c & 4 \\
\hline d & 40 & $\begin{array}{ll}0 & 4\end{array}$ & $\mathrm{~d}$ & 4 & 4 & 4 & $\mathrm{~d}$ & 4 \\
\hline
\end{tabular}
each column.

Since we wish to minimize the maximum regret $\mathrm{MR}$, the optimal alternative w.r.t. minimax regret is therefore $a$ when considering non-increasing weights.

Now, note that, since $\frac{\mathcal{V}_{j}^{x}}{j}$ represent the score associated to the top-j Borda rule, Equation 15 basically states that the computation of max regret when $w$ lies in $W^{C}$ is equivalent to consider the loss occurred by $x$ with respect to the family of top-k Borda aggregators (that includes plurality and Borda as a special case).

Example 5. We consider again the running example and compute the max regret values when considering that the scoring vector is a convex sequence, using Equation 15. First, we compute the double cumulative distribution of the ranks; we then divide the second column by two, the third by three, etc. Then, for computing the maximum regret of $a$, we consider the maximum, among columns, between the value of $a$ and the best (bold) values, i.e. $\max \{4-2,4-3,4.66-4\}=$ 2 . The minimax regret optimal candidate in this case is therefore $d$.

\footnotetext{
${ }^{4}$ Proofs are available from the author upon request.
} 


\begin{tabular}{lcccccc}
\hline Candidate & $\mathcal{V}_{1}$ & $\frac{\mathcal{V}_{2}}{2}$ & $\frac{\mathcal{V}_{3}}{3}$ & & & \multicolumn{2}{c}{ Candidate $\mathrm{MR}_{W^{C}}$} \\
\hline $\mathrm{a}$ & 2 & 3 & 4 & & $\mathrm{a}$ & 2 \\
$\mathrm{~b}$ & 0 & 3 & $\mathbf{4 . 6 6}$ & & $\mathrm{b}$ & 4 \\
$\mathrm{c}$ & 2 & 2 & 3.33 & & $\mathrm{c}$ & 2 \\
$\mathrm{~d}$ & $\mathbf{4}$ & $\mathbf{4}$ & 4 & & $\mathrm{~d}$ & $\mathbf{0 . 6 6}$ \\
\hline
\end{tabular}

Expected score Assuming a distribution over the weights, it is possible to rank alternatives by the expected score. Let $P(w)$ be the such distribution. By the linearity of expectation, we can sort alternatives by using a scoring rules using the expected values of the uncertain weights: $\mathbb{E}_{w \sim P(w)}\left[s_{w}(x)\right]=\sum_{j=1}^{m} \mathbb{E}_{w \sim P(w)}\left[w_{j}\right] v_{j}^{x}$. Therefore it means that sorting the candidates by expected score - under distribution $P(w)$ - is equivalent to using a scoring rule whose weights are given by $\mathbb{E}\left[w_{j}\right]$, the expectations of the weights. The choice of the probability distribution over the weights is critical; we observe that if we assume an uniform distribution over non-increasing weights, sorting by expectation is equivalent to Borda.

Proposition 4 Assume $w$ uniformly distributed in $W^{D}$ (non-increasing weights)

$$
\mathbb{E}_{w \sim U}\left[s_{w}(x)\right]=s_{\text {Borda }}(x)
$$

For convex sequences, we don't have a closed formula for the expected weights in $W^{C}$. We rely on Monte Carlo methods based on Gibbs sampling to derive numerical values for the expected weights. In Figure 1 we show the weights obtained by using expectation assuming an uniform distribution over all possible convex sequences. In the plot we compare the expected weights with that (normalized) used in official F1 car races (that adopt a fixed convex sequence). The F1 weights (dashed lines) can be considered rather "steep" since they only award points to a small number of top drivers while often there are about 15-20 drivers (cfr with expected weights with $m=22$, in light blue); but if one wishes to award points only to the best 8 or 9 drivers, then the F1 weights are actually less "steep" than the ones obtained by expectation over convex sequence (in the plot consider the line representing $m=9$, in violet).

\section{Numerical Tests}

We consider the rankings of the F1 race championship from 1961 to $2008^{5}$. In this context, a candidate is a driver and each ranking is the result of a race. In Table 1 below, we show the number of undominated alternatives and the number of possible winners in the case of monotone weights and that of convex weights. Several alternatives are Pareto dominated (e.g. a driver is Pareto dominated if there is another one that is better ranked in all races); the number of Pareto candidates is shown on the fourth column (for instance 16 drivers out of 54 are Pareto in 1961, while 17 out 22 in 2008).

\footnotetext{
${ }^{5}$ Obtained from the PREFLIB data repository (http://www.preflib.org/).
} 


\begin{tabular}{|c|c|c|c|c|c|c|c|}
\hline \multirow{2}{*}{$\frac{\text { dataset }}{1961}$} & \multirow{2}{*}{$\frac{\mathrm{m}}{54}$} & \multicolumn{2}{|c|}{ n Pareto } & \multicolumn{2}{|c|}{$\begin{array}{c}\text { Monotone } \\
\text { \#Und. \#P.W. }\end{array}$} & \multicolumn{2}{|c|}{$\frac{\text { Convex }}{\text { \#Und. \#P.W. }}$} \\
\hline & & 8 & 16 & 3 & 3 & 2 & 2 \\
\hline 1962 & 51 & 9 & 12 & 1 & 1 & 1 & 1 \\
\hline 1963 & 54 & & 8 & 1 & 1 & 1 & 1 \\
\hline 1964 & 42 & 10 & 17 & 6 & 5 & 4 & 4 \\
\hline 1965 & 52 & 10 & 13 & 2 & 2 & 2 & 2 \\
\hline 1966 & 43 & 9 & 20 & 4 & 4 & 2 & 2 \\
\hline 1967 & 47 & 11 & 14 & 4 & 4 & 3 & 3 \\
\hline 1968 & 44 & 12 & 22 & 4 & 4 & 2 & 2 \\
\hline 1969 & 42 & 11 & 15 & 4 & 4 & 1 & 1 \\
\hline 1970 & 43 & 13 & 21 & 8 & 8 & 5 & 4 \\
\hline 1971 & 50 & 11 & 21 & 4 & 4 & 1 & 1 \\
\hline 1972 & 44 & 12 & 25 & 7 & 7 & 1 & 1 \\
\hline 1973 & 44 & 15 & 19 & 4 & 3 & 1 & 1 \\
\hline 1974 & 62 & 15 & 23 & 3 & 3 & 2 & 2 \\
\hline 1975 & 52 & 14 & 22 & 6 & 6 & 1 & 1 \\
\hline 1976 & 57 & 16 & 23 & 6 & 6 & 3 & 3 \\
\hline 1977 & 61 & 17 & 26 & 5 & 5 & 5 & 4 \\
\hline 1978 & 49 & 16 & 26 & 6 & 6 & 1 & 1 \\
\hline 1979 & 36 & 15 & 20 & 3 & 3 & 3 & 3 \\
\hline 1980 & 41 & 14 & 23 & 2 & 2 & 1 & 1 \\
\hline 1981 & 40 & 15 & 22 & 5 & 5 & 3 & 3 \\
\hline 1982 & 40 & 16 & 26 & 6 & 6 & 4 & 4 \\
\hline 1983 & 35 & 15 & 26 & 3 & 3 & 2 & 2 \\
\hline 1984 & 35 & 16 & 24 & 3 & 3 & 2 & 2 \\
\hline 1985 & 36 & 16 & 24 & 3 & 3 & 1 & 1 \\
\hline 1986 & 32 & 16 & 20 & 4 & 4 & 2 & 2 \\
\hline 1987 & 32 & 16 & 25 & 8 & 8 & 2 & 2 \\
\hline 1988 & 36 & 16 & 16 & 2 & 2 & 2 & 2 \\
\hline 1989 & 47 & 16 & 30 & 5 & 5 & 2 & 2 \\
\hline 1990 & 40 & 16 & 20 & 5 & 5 & 3 & 3 \\
\hline 1991 & 41 & 16 & 19 & 1 & 1 & 1 & 1 \\
\hline 1992 & 39 & 16 & 23 & 5 & 5 & 1 & 1 \\
\hline 1993 & 35 & 16 & 24 & 1 & 1 & 1 & 1 \\
\hline 1994 & 46 & 16 & 24 & 4 & 4 & 2 & 2 \\
\hline 1995 & 35 & 17 & 26 & 6 & 6 & 1 & 1 \\
\hline 1996 & 24 & 16 & 20 & 2 & 2 & 1 & 1 \\
\hline 1997 & 28 & 17 & 22 & 4 & 4 & 2 & 2 \\
\hline 1998 & 23 & 16 & 17 & 3 & 3 & 2 & 2 \\
\hline 1999 & 24 & 16 & 18 & 2 & 2 & 2 & 2 \\
\hline 2000 & 23 & 17 & 21 & 4 & 4 & 2 & 2 \\
\hline 2001 & 26 & 17 & 19 & 2 & 2 & 1 & 1 \\
\hline 2002 & 23 & 17 & 5 & 1 & 1 & 1 & 1 \\
\hline 2003 & 24 & 16 & 16 & 3 & 3 & 2 & 2 \\
\hline 2004 & 25 & 18 & 16 & 2 & 2 & 1 & 1 \\
\hline 2005 & 27 & 19 & 19 & 2 & 2 & 1 & 1 \\
\hline 2006 & 27 & 18 & 19 & 2 & 2 & 1 & 1 \\
\hline 2007 & 26 & 17 & 17 & 4 & 4 & 3 & 3 \\
\hline 2008 & 22 & 18 & 17 & 4 & 4 & 2 & 2 \\
\hline
\end{tabular}

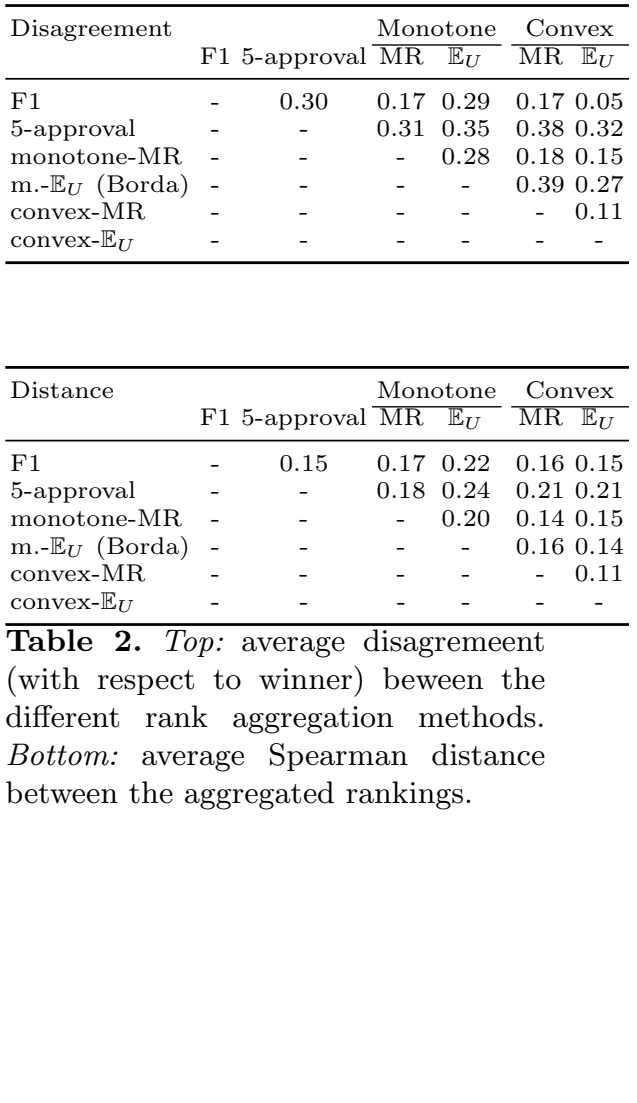

Table 1. Number of undominated items and possible winners in actual rank data. (Legend: \#Und.=number of undominated, \#P.W.:=number of possible winners, $m=$ number of drivers, $n=$ number of races). 


\begin{tabular}{lrrrrrrrrrr}
\hline $\mathrm{m}$ & $w_{1}$ & $w_{2}$ & $w_{3}$ & $w_{4}$ & $w_{5}$ & $w_{6}$ & $w_{7}$ & $w_{8}$ & $w_{9}$ & $w_{10}$ \\
\hline 3 & 1.00 & 0.25 & 0.00 & - & - & - & - & - & - & - \\
4 & 1.00 & 0.39 & 0.12 & 0.00 & - & - & - & - & - & - \\
5 & 1.00 & 0.47 & 0.21 & 0.07 & 0.00 & - & - & - & - & - \\
6 & 1.00 & 0.55 & 0.29 & 0.13 & 0.04 & 0.00 & - & - & - & - \\
7 & 1.00 & 0.57 & 0.32 & 0.17 & 0.08 & 0.02 & 0.00 & - & - & - \\
8 & 1.00 & 0.61 & 0.39 & 0.23 & 0.13 & 0.06 & 0.02 & 0.00 & - & - \\
9 & 1.00 & 0.64 & 0.42 & 0.27 & 0.16 & 0.09 & 0.04 & 0.01 & 0.00 & - \\
10 & 1.00 & 0.69 & 0.48 & 0.33 & 0.21 & 0.13 & 0.07 & 0.03 & 0.01 & 0.00 \\
\hline
\end{tabular}

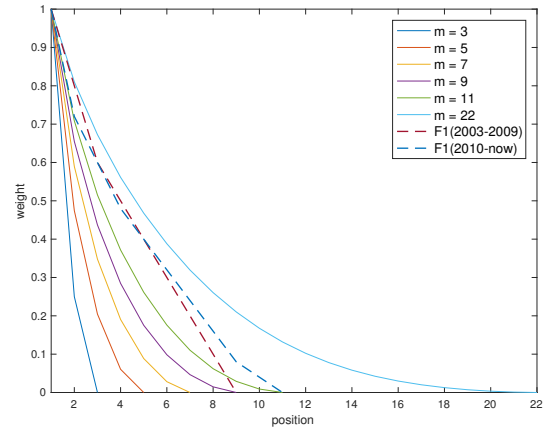

Fig. 1. Table (on the left): weights obtained by uniformly sampling a convex sequence of $m$ positions; plot (right): sampled weights with the weights used by F1 races.

We then analyze (using the propositions of Section 2) which drivers are undominated, and which are possible winners (Section 2.3). For monotone scoring vectors, there are often several possible winners but never more than 8 . In just 5 years we have a necessary winner $(1962,1963,1991,1993,2002)$, meaning that setting the scoring vector correctly is critical. When assuming convex scoring vectors, the number of possible winners is considerably reduced (and there is a necessary winner in several circumstances). Note that, although not frequently, there are sometimes undominated candidates that are not possible winners.

In Table 2 (top) we analyze how often the different rank aggregation methods (including ranking with the $\mathrm{F} 1$ point system and k-approval) disagree ${ }^{6}$ on picking the winner; while most of the time they pick the same winners, there is a considerable difference between. There is a considerable disagreement between Borda and the expected score assuming convexity (more than 1 out of 4 times the winner is different), and as well between Borda and convex MR. Since scoring rules can also be used to produce rankings, we also compare (Table 2 bottom) the rankings obtained by the different methods (e.g. sorting by ascending MR scores) using the Spearman distance.

\section{Conclusions}

In this article we have considered preference aggregation in the context of social choice when the scoring vectors are not defined apriori. The fact that using scoring rules with different weights may produce very different outputs have been noticed before; some of these methods are reviewed in [7]. In this paper we first considered the problem of discriminating which alternatives are possible winners, and the considered aggregation methods motivated by decision-theory (in particular, minimax regret and expected score).

\footnotetext{
6 To handle the case of ties (when a method returns multiple winners) we compute disagreement as the cardinality of the symmetric set difference normalized by the cardinality of the union.
} 
In this paper we take the assumption that a committee specifies a generic settings for the scoring vector, that is using monotone or convex sequences; we believe that this is quite reasonable if one wish to use our proposed methods as social choice function. For minimax regret, the computation is quite easy using the cumulative (and double cumulative) ranks, therefore one could adopt such technique as an aggregation method. One could of course add additional desiderata about the weights or even consider elicitation protocols - in that case one would need to compute regret using optimization tools [12].

Note that the provided methods could be also used with an informative goal: for instance, for inspecting which candidates are possible winners (letting the decision maker discriminate between such alternatives in a second moment). The fact that the expectation of a uniformly distributed decreasing sequence gives the weights that are linearly spaced (as Borda), can be seen as an additional theoretical justification for using Borda.

\section{References}

1. Nawal Benabbou and Patrice Perny. Incremental weight elicitation for multiobjective state space search. In Proc. of AAAI, pages 1093-1099, 2015.

2. C. Boutilier, R. Patrascu, P. Poupart, and D. Schuurmans. Constraint-based Optimization and Utility Elicitation using the Minimax Decision Criterion. Artifical Intelligence, 170(8-9):686-713, 2006.

3. Wade D. Cook and Moshe Kress. A data envelopment model for aggregating preference rankings. Management Science, 36(11):1302-1310, 1990.

4. Peter Fishburn and William Gehrlein. Borda's rule, positional voting, and Condorcet's simple majority principle. Public Choice, 28:79-88, 1976.

5. Judy Goldsmith, Jérôme Lang, Nicholas Mattei, and Patrice Perny. Voting with rank dependent scoring rules. In Proc. of AAAI, pages 698-704, 2014.

6. P. Kouvelis and G. Yu. Robust Discrete Optimization and Its Applications. Kluwer, Dordrecht, 1997.

7. Bonifacio Llamazares and Teresa Peña. Preference aggregation and DEA: an analysis of the methods proposed to discriminate efficient candidates. European Journal of Operational Research, 197(2):714-721, 2009.

8. Tyler $\mathrm{Lu}$ and Craig Boutilier. Robust approximation and incremental elicitation in voting protocols. In Proceedings of IJCAI 2011, pages 287-293, 2011.

9. A. Salo and R. P. Hämäläinen. Preference ratios in multiattribute evaluation (PRIME)-elicitation and decision procedures under incomplete information. IEEE Trans. on Systems, Man and Cybernetics, 31(6):533-545, 2001.

10. L. J. Savage. The Foundations of Statistics. Wiley, New York, 1954.

11. William E. Stein, Philip J. Mizzi, and Roger C. Pfaffenberger. A stochastic dominance analysis of ranked voting systems with scoring. European Journal of Operational Research, 74(1):78 - 85, 1994.

12. Paolo Viappiani and Craig Boutilier. Regret-based optimal recommendation sets in conversational recommender systems. In Proc. of ACM RecSys, pages 101-108, 2009.

13. H. P. Young. An axiomatization of borda's rule. Journal of Economic Theory, 9:43-52, 1974.

14. H. P. Young. Social choice scoring functions. SIAM Journal on Applied Mathematics, 28(4):824-838, 1975. 